\title{
Study on Knowledge Propagation in Complex Networks Based on Preferences, Taking Wechat as Example
}

\author{
Si-hua Chen ${ }^{1,2}$ and Wei He \\ ${ }^{1}$ School of Information Technology, Jiangxi University of Finance and Economics, No. 169, East Shuanggang Road, Changbei, \\ Nanchang, Jiangxi 330013, China \\ ${ }^{2}$ Jiangxi Key Laboratory of Data and Knowledge Engineering, Jiangxi University of Finance and Economics, Nanchang 330013, China \\ ${ }^{3}$ School of Business and Administration, Jiangxi University of Finance and Economics, No. 169, East Shuanggang Road, Changbei, \\ Nanchang, Jiangxi 330013, China
}

Correspondence should be addressed to Si-hua Chen; doriancsh@foxmail.com

Received 11 April 2014; Revised 29 June 2014; Accepted 7 July 2014; Published 23 July 2014

Academic Editor: Zidong Wang

Copyright (c) 2014 S.-h. Chen and W. He. This is an open access article distributed under the Creative Commons Attribution License, which permits unrestricted use, distribution, and reproduction in any medium, provided the original work is properly cited.

As platform based on users' relationship to acquire, share, and propagate knowledge, Wechat develops very rapidly and becomes an important channel to spread knowledge. This new way to propagate knowledge is quite different from the traditional media way which enables knowledge to be spread surprisingly in Wechat. Based on complex network theory and the analysis of the factors which influence the knowledge propagation in Wechat, this paper summarizes the behavior preferences of Wechat users in knowledge propagation and establishes a Wechat knowledge propagation model. By the simulation experiment, this paper tests the model established and finds some important thresholds in knowledge propagation in Wechat. The findings are valuable for further studying the knowledge propagation in Wechat and provide theoretical proof for forecasting the scale and influence of knowledge propagation.

\section{Introduction}

Since 1990s with the rapid development of global information technologies, global economy is endowed with the informationized, cyberized, and knowledge-focused features. These features turn the global economy from material-based economic activities to knowledge-based economic activities [1]. Consequently, knowledge management becomes one of the hot issues for both managerial theorists and practice experts since 2001 [2]. In the fast changing new economy environment, the quick acquisition and creation of knowledge as well as the ability to utilize knowledge are no doubt the keys for an organization to obtain sustainable competitive advantage. The traditional organizational forms and management modes featured as labor of division, hierarchy, and clear functional boundary are facing great challenges. The networks of organizations built on knowledge are gradually becoming the new practices in economic and managerial field. In knowledge economy, the social networks among employees not only change the traditional ways to produce and organize but also continuously reshape the internal and external environment where an organization lives. All these pose severe challenges for both managers and researchers. The employee knowledge is regarded as development foundation of an organization. Knowledge is power while the propagation of knowledge is the extension of such power. Based on theoretical research and managerial practices, it is found that the employee knowledge is embedded in the connections of employees [3-6]. The sharing and propagation of employee knowledge are a complex activity process $[7,8]$. In the current "social networking" trend, these characteristics of employee knowledge inevitably make great impact on traditional knowledge management and pose brand new requirements for the sharing of employee knowledge within complex social networks.

From practical perspective the knowledge propagation is always a problem for managers. Because of the different culture backgrounds the determinants of the sharing 
of employee knowledge vary a lot $[9,10]$. Basically the knowledge propagation means the process in which individuals teach other individuals, teams, and knowledge bases with their own professional knowledge, ideas, or experiences so that the receivers can master the knowledge to the greatest extent. Usually the process happens in certain organizational context and is embedded in a giant social network. As integrated system of social relationships and technical needs, through individuals' motive sequence and subjective mechanism, the complex social network can influence individuals' behavior selection. Under such practical background, to effectively solve the problem of employee knowledge sharing and fully make use of the social network to reduce the cost of knowledge sharing and further improve the knowledge utilization efficiency, it is very necessary to clarify the behavior logic behind the employee knowledge propagation in complex social networks and master the law of strategy selection of which complex social networks influence employee knowledge propagation behaviors. And this becomes the practical problem in current knowledge management waiting to be solved.

Knowledge propagation is the precondition of knowledge innovation and knowledge utilization. If there is no knowledge propagation, the utility of knowledge will be only confined to individuals $[11,12]$. Therefore, knowledge needs to continuously flow and be transferred and then it can display its maximum utility [13]. Knowledge management can produce value mainly through the acquisition, creation, classification, storage, propagation, and renewal of knowledge. During the consecutive processes, the most difficult problem is the knowledge propagation. Hence, knowledge propagation is a very important issue in knowledge management.

Until now there is no agreement on the definition of knowledge propagation. Scholars in different fields propose different views on knowledge propagation behaviors: from communication perspective, knowledge propagation is a communication process [14], which is inevitably involved in the cognition of both sides in communication. Thereby, knowledge propagation has two subjects, that is, the knowledge owners and the knowledge receivers. Usually the owners and the receivers have common experiences which are the key factor for the successful transfer. In addition, some scholars try to explain knowledge propagation from the knowledge market perspective. They reckon the knowledge itself is influenced by the supply and demand law. Knowledge is regarded as transacting object just like the commodities. There exists a kind of relationship between the supply and the demand, that is, the knowledge seller and the knowledge buyer. They transact to obtain what they need. Such kind of transaction is successful because both sides of the transaction think they can get benefits from it. And this is the power source for the knowledge sharing. Thereby, in practice enterprises should fully understand and make use of knowledge market mechanism and provide incentives to encourage knowledge sharing. In these ways knowledge transaction can be greatly promoted $[15,16]$. Extending from the market view is the view which regards knowledge propagation as social exchange mechanism. Some scholars hold the view that knowledge propagation behaviors are a kind of social exchange. Economic exchange depends on the formal contracting between the two sides which formulates the transactional quantities and responsibilities in detail. Different from the economic exchange, the social exchange view thinks the mutual reciprocity and trust between both sides of the knowledge transfer are the main factors which influence the knowledge sharing. Although knowledge sharing is similar with the transaction of commodities and services, it is more to think both sides in the knowledge propagation have the social exchange mindset than to say the knowledge propagation possesses the transactional features [17-19]. Apart from the communication view, market view, and the social exchange view, there are scholars who regard knowledge propagation as one of the learning modes. Knowledge propagation is the ability to help others to develop effective behaviors and learn about the reasons of things. Knowledge propagation is the "teaching" activity of knowledge owners. For the knowledge demander, knowledge propagation is a kind of "learning" activity [20-22].

From the above literature review, the author finds the studies on knowledge propagation are mainly based on three theoretical perspectives.

(1) The Communication Perspective. This perspective intends to explore the general law of knowledge propagation under the macrosocial background. Most of these studies are involved in discussing the problems such as the speed, obstacles, and characteristics of knowledge propagation. The virus spreading model is the classical propagation dynamics model. Its basic thought is to classify the individuals according to certain rule and then according to the shift of types of different individuals caused by the interactions in knowledge propagation to establish the mathematical model [23-25]. The virus spreading model mainly focuses on the speed and degree of knowledge propagation. The classical propagation models include SI, SIR, and SIS models.

(2) The Organizational Perspective. This perspective concentrates on problems such as motivation and willingness to propagate knowledge. It thinks there are two kinds of determinants which may lead to the happening of knowledge propagation: the determinants from psychological, social, and technical aspects and the determinants from behavioral aspect. The former one includes organizational incentives, mutual benefits, improvement of position, readiness to help others, organizational atmosphere, tools, and techniques; the latter one includes the attitude to knowledge sharing, subjective norms, and behavior controlling. The knowledge propagation behaviors are influenced by three groups of forces: the balance between costs and benefits, the demand differences of the allocation of sharing outcomes, and the structured relationship of the knowledge receivers [26-28].

(3) The Complex Network Perspective. The complex network theory is used to explain the complexity problem in real world. Currently only very few scholars tried to use the complex network models to analyze the network structure and function of knowledge propagation and innovation 
diffusion. The most influential application is the study made by Rogers on innovation diffusion [29]. Cowan et al. examined the influence of network structure such as regular network, random network, and small world network on knowledge diffusion between individuals. They found that the knowledge diffusion in small world network was the fastest [30, 31]. Morone and Taylor (2004) proposed a rule to test the knowledge propagation in a general faceto-face network [32]. Jianxun (2006) modified the model of Cowan and took the unexplored problem into consideration [33]. Separately from the macro- and microlevel of structure dynamics and behavior dynamics of knowledge propagation, he constructed a knowledge propagation dynamics model based on complex network to systematically discuss the dynamic evolutionary problem of knowledge propagation based on complex network. Aiming at the spreading characteristics of knowledge in knowledge cooperation network, combined with the complex network theory, $\mathrm{Li}$ and Sun (2006) proposed a knowledge propagation model in complex networks. They thought within a group the increase of the knowledge of an individual relied on the individual's effort and the knowledge spillover effect brought by the nearest individuals in the neighborhood [34]. By applying the complex network theory and organizational simulation, Wanly (2008) found that knowledge propagation was closely related to the spreading speed of knowledge in organization, the success probability, and the attitudes of knowledge owners [35].

At present the studies on knowledge propagation in complex networks are based on the perspectives of network connection and network structure.

Scholars divide the complex network connections into strong connections and weak connections. Furthermore, they examine the influence of different connections on knowledge propagation and the interactions between strong connections and weak connections. The empirical studies show when knowledge is not complex, the weak connections can accelerate the knowledge acquisition. If the knowledge is complex, the weak connections may reduce the transfer. Individuals tend to have strong connections or they will share the knowledge with individuals who are perceived with good relationship [36, 37]. For the knowledge propagation in the internal network of an organization, scholars think the unconnected relationship between employees of the organization is not good to the speed and scale of knowledge propagation in the organization. However, the few repeated weak connections between employees of the organization can accelerate the speed of knowledge propagation and enlarge the scale of knowledge propagation through the "intermediate" connections [38].

Some other scholars focus their studies on the structure of social network. They think comparing with social network connections, the social network structure can better explain the knowledge sharing function of social network. The willingness of individuals of an organization to put time and efforts in the knowledge sharing is influenced by the social cohesion. For different types of knowledge, the knowledge sharing between individuals and groups is also influenced by the network scale. From the network structure dimensions such as network density, centrality, and structure holes scholars have explored the influence of network structure on the quality, the timing, and scale of knowledge propagation $[39,40]$.

According to the above literature review, we can find the discussions of the social network perspective on the influence of social network on individual or organizational knowledge propagation are separate from the social network connection aspect (such as connection strength, network density, and network scale) and network structure aspect (such as bridge connection and structure hole). These studies have made great contributions to the knowledge propagation field and at the same time have laid a solid foundation for the future studies. However, because it is a new research field which develops very quickly, there are inevitably some shortcomings and they are as follows.

(1) Currently most of the complex network based knowledge propagation studies are built on the classical epidemic models such as the simulation study of rumor spreading in microblogging community [41]. By taking the advantage of the similarity of the spreading of rumors with that of virus, the study explored the propagation mechanism of rumors in network from macroaspect. However, for knowledge propagation, not only the propagation activity will attenuate quickly with time because different connections have different spreading ability and spreading modes but also knowledge propagation is influenced by the spreading content. The studies on these problems are still rare.

(2) The knowledge propagation in complex networks can be attributed to the transmitting behaviors of knowledge subjects. But the knowledge subjects are diversified and their transmitting behaviors are related to their historical behaviors. Currently most studies only analyze the knowledge propagation from the network structure and connection perspectives and seldom take the heterogeneity of knowledge subjects into consideration. This provides further study space for this paper.

Wechat is a free applied program launched by Tencent enterprise in January 21, 2011, which provides instant messaging services for intelligent phones. According to the statistics of Baidu encyclopedia, until April 152013 the number of registered users of Wechat (the abroad edition) has broken through 10 million. Within just a month, the users have increased 3 million. Until October 24, 2013, the number of users of Tencent Wechat has exceeded 60 million and the daily active users are about 10 million (from Baidu encyclopedia).

What Wechat propagates is quite private and the propagation is instant. The social circle of Wechat is mainly comprised of relatives, friends, and colleagues. Due to the special relationship between senders and receivers, what is propagated in Wechat is quite private. On the Wechat, fans can see the related information released by the users they are following with interest. The Wechat information is on the 
mobile terminals of senders and receivers and only both of them can see or hear. Other users cannot obtain the information on their own. What is more, Wechat has integrated the functions of QQ and microblog. Its information release is instant. As long as the users are on the line, they can quickly receive and feedback the information. Besides, Wechat can support the receiving of QQ off line message. It is swift in message sending and receiving. Apart from that, the users of Wechat even can show their present state or make comments by Chinese characters, pictures, or display of geographical location. Other users in the friend circle can instantly release their comments. In these ways, Wechat can satisfy people's personal expression in the personalized Era. Some of the content propagated on Wechat is just describing personal state and emotional information (such as traveling places, pictures of eating, or personal feelings). The probability of this information being retweeted is small; some of the content is about know what, know how, know who, and know why. The probability of being retweeted is relatively big. What this paper focuses on is knowledge propagation on Wechat.

Relying on Wechat network platform, this paper studies the knowledge propagation behaviors of different knowledge subjects who have different preferences. Based on the analysis of the speed, scale, and scope of knowledge propagation, this paper summarizes the knowledge propagation path and the law.

This paper proceeds as follows: the second part analyzes the preferences of Wechat users; the third part establishes the knowledge propagation model; the fourth part makes a simulation experiment and analyzes the result. Based on it, this part draws a conclusion.

\section{Analysis of the Preferences of Knowledge Propagation Subjects}

Preference is an important issue in artificial intelligence. In recent years studies on preference have attracted lots of attention from both domestic and abroad journals. Currently preferences are described based on CP-nets. By discussing the factors which influence users' knowledge propagation behaviors in Wechat, this paper applies the CP-nets preference expression tool to establish preference model of users' transmission behaviors.

2.1. Factors Influencing the Knowledge Propagation. By literature review, the factors which influence the knowledge propagation in networks generally include the users active degree, the importance of content, the interest of users, and the common interests between users [42, 43].

The users active degree reflects the characteristics of an individual user's behavior. Users can choose to retweet the knowledge they are interested in or just read and not to spread the knowledge. Yang et al. found statistically that the retweeting frequencies of most users are low. Only very small number of users is keen on retweeting messages. Apart from that, the users active degree is also related to the users' release and reply behaviors. By calculating on the retweeting number of a user within certain time we can make judgment on whether the user is a retweeting fan and therefore help to forecast the user's retweeting decision. If the user is a retweeting fan, the user will to great degree retweet the messages while not caring about the content of these messages and the senders. If the user is just a common user, we need to use more factors to measure whether he or she will retweet.

The content of messages can be regarded as driving force originated from the inside of the information for the knowledge propagation in Wechat community. Generally speaking, the hot topics are more often referred to in the community while the minority interests are only discussed by particular users. This phenomenon shows the importance of the content of messages. By calculating the frequencies of the words used by a user in his or her Wechat within certain time, we can evaluate the importance of the original content of Wechat. We use the sum of the TF-IDF value of each keyword in the original content to denote the importance of original content.

From a user's interest in the content of Wechat, we can see that the extent of a user's interest in the message can clearly show whether they will retweet or ignore the message. It can exert great influence on a user's decision. As individuals to release the Wechat messages, the relationship between the person and the message is closer than that between any propagator and the message. When a user is more interested in the content of a Wechat message, the common interest of the user and the person who releases the message can be an active factor influencing the user's retweeting decision.

The users' selections are also influenced by the knowledge propagators who have the same interests. The common interests of users mainly include the common interests between the users and the persons who release the messages, the common interests between users and the nearest propagators, and the common interests between the users and other propagators. Studies showed although the users could retweet knowledge from the nonneighbor propagators, the users still mainly acquire knowledge and retweet the knowledge from their nearest propagators.

\subsection{The Preference Expression of Knowledge Propagation.} Based on the above analysis, this paper applies the paired comparison method to analyze the preferences of Wechat users. We design the paired comparison scales and select 800 Wechat users from 50 Wechat groups of undergraduates in Jiangxi University of Finance and Economics to fill the questionnaires. We received 178 effective questionnaires. By processing the data, we have the following results.

(1) When receiving new knowledge, $84 \%$ students attach more importance to the knowledge content rather than the propagation path.

(2) For students who pay lots of attention to knowledge content, $91 \%$ of them pay more attention to the similarity of content with their own interests.

(3) For students who pay lots of attention to propagation path, $81 \%$ of them pay more attention to the similarity of knowledge propagators with their own interests. 
(4) For students who pay lots of attention to content and their own interests, $78 \%$ of them pay more attention to the similarity of the persons who release the messages and their own interests.

(5) For students who pay lots of attention to knowledge propagators and their own interests, $87 \%$ of them pay more attention to the similarity of their nearest knowledge propagators and their own interests.

According to the above results, we can establish the $\mathrm{CP}$-nets expression of the knowledge propagation preferences of Wechat users. The preference expression $\mathrm{CPT}(A)$ corresponds to (1); CPT $(B)$ corresponds to (2) and (3); $\mathrm{CPT}(C)$ corresponds to (4) and (5). A denotes knowledge, $A_{t}$ denotes knowledge content, $A_{r}$ denotes propagation path; $B$ denotes interests, $B_{t}$ denotes the interests in content, $B_{r}$ denotes the interests in propagators; $C$ denotes the common interests between users, $C_{t}$ denotes the common interests with the persons who release messages, $C_{r}$ denotes the common interests with the nearest propagators; based on the relationship between preferences we can get the users' preference expression map.

From the preference expression map we can infer the following four preference sequences:

$$
\begin{gathered}
A_{t} B_{t} C_{t}>A_{t} B_{t} C_{r}>A_{r} B_{t} C_{r} \\
A_{t} B_{t} C_{t}>A_{r} B_{t} C_{t}>A_{r} B_{t} C_{r} \\
A_{t} B_{t} C_{t}>A_{t} B_{r} C_{r}>A_{t} B_{r} C_{t}>A_{r} B_{t} C_{t}>A_{r} B_{t} C_{t} \\
>A_{r} B_{t} C_{r} A_{t} B_{t} C_{t}>A_{t} B_{r} C_{r}>A_{r} B_{r} C_{r} \\
>A_{r} B_{r} C_{t}>A_{r} B_{t} C_{t}>A_{r} B_{t} C_{r} .
\end{gathered}
$$

From it we can see the best output is $A_{t} B_{t} C_{t}$. That is, when users prefer the original message content, we select the importance of the original content, the Jaccard similarity between users' interests and content, the Jaccard similarity between users' interest and the persons' interests who release messages as key factors to evaluate whether the user will make the retweeting decision after receiving the message. Based on it, when evaluating the decisions, we only need to use the characteristic value of the three feature items.

\section{Wechat Knowledge Propagation Model}

As typical network application in Web 2.0, mainly through large amount of knowledge produced by users' behaviors Wechat can attract more users to experience. This study on the knowledge propagation in Wechat community mainly focuses on the word knowledge released by users.

3.1. Analysis on Users' Propagation Decisions. To study the knowledge propagation mechanism in Wechat community, this paper defines the users in Wechat community and the knowledge propagated as Agent. Based on the "friend circle" relationship network among users in community, according to the individual's properties and the interactive rules we construct the knowledge propagation network. By taking advantage of users' historical behavior information and preferences we can forecast the trend of knowledge propagation and analyze the simulation results. Suppose user $A$ receives the knowledge $K$ retweeted from user $B$. Then how does user $A$ retweet this knowledge? How to measure the user's retweeting behavior? We will analyze the decision process.

Scholars have divided the individuals in a social network based on their behaviors. Basically users in Wechat community can be classified into three types: readers who intend to read, writers who intend to write, and editors who intend to propagate. Furthermore, the propagation of knowledge in network is closely related to the content: some events happen suddenly such as some news topics which have strong timeliness while some events are discussed continuously such as some technical or life related topics. Thereby, we divide the events the content is involved in into two types: the sudden events and common events.

To make judgment on whether user $A$ will retweet the knowledge, first we need to know the types of users (User_type). Based on their behaviors, we can divide users in Wechat into readers (User_type $=0$ ) who only browse and not comment and retweet messages; writers (User_type = 1) who usually release the original Wechat knowledge and not retweet the Wechat knowledge they receive; editors (User_type $=2$ ) who prefer to retweet messages they receive. If user $A$ is a reader or a writer, usually user $A$ will not retweet the knowledge $K$. Otherwise, if user $A$ is an editor, he or she will need to make judgment on the types of the events (Infor_type). When it is an important sudden event (Infor_type $=1$ ) rather than a common event (Infor_type = 0 ), user $A$ will retweet the Wechat message. Otherwise, we need to make further judgment.

Based on the above analysis of the factors which influence a users' decision, We can consider all the factors such as the importance of the original content (Infor_importance), the Jaccard similarity between users' interest and content (Jaccard_user_infor), the Jaccard similarity between users' interests and the persons' interests who release messages (Jaccard_user_ini), and the Jaccard similarity between users' interests and the nearest propagators' interests (Jaccard_user_con). These factors interact with each other. The above section has analyzed them based on preferences. By selecting the best feature item, we simplify the complexity to compute the characteristic values which influence the users' decision behaviors. The specific procedure is shown in Algorithm 1.

The basic thought of the algorithm is to first deal with the extreme conditions such as the sudden events or extreme active users and then to measure the users' retweeting behaviors in common conditions. It generalizes the whole decision process of individual users to retweet knowledge. In the following part we will mainly discuss the formation process of a knowledge propagation network based on the retweeting behavior of an individual user.

Currently most of the studies on knowledge propagation are built on the classical epidemic models. And most of them are the Poisson model or the critical value model. 
Input: User $A$ and Knowledge $K$

Output: degree of preference of User $A$ to retweet Knowledge $K$

Step 1 .

initiate User $A$, Knowledge $K$;

// initiate User $A$, obtaining User $A$ 's historical behavioral information (the number of releasing messages (num), the number of retweeting messages (num 1)) and the persons who release messages in Wechat $K$, the nearest propagators and the original

content ect.;

Step 2.

make judgment on the type of User $A$ and the type of events

// make judgment on the type of User A (User_type) and the type of events involved in Wechat (Infor_type)

Step 3.

Based on the type of the user and the type of events we can judge whether it satisfies the end condition;

// If User_type $=0 \vee$ User_type $=1$, we judge the user will not retweet the message and the algorithm ends; If User_type $=2$ and Infor_type $=1$, we judge the user will retweet the message and the algorithm ends; If User_type $=2$ and Infor_type $=0$, then we need further judgment and enter the fourth step;

Step 4.

If it does not satisfy the end condition, we need to select the best feature item combination to further evaluate;

// select the best feature item combination and attribute it to the decision preference set

Step 5 .

calculate the characteristic value of the feature item selected

// obtain the value of the feature item

Step 6.

obtain the degree of preference of User $A$ to retweet Knowledge $K$

// Calculate the degree of retweeting preference of the user

Algorithm 1

However, not only the propagating activity in knowledge propagation will attenuate with time but also the different types of propagation have different propagation power and propagation modes. In addition, the knowledge propagation is greatly influenced by the content. Different knowledge will activate different propagation network. In Wechat the knowledge propagation is attributed to the retweeting behaviors of users. And the knowledge propagation in Wechat has many uncertainties and is related to users' historical behaviors. Therefore, we cannot adopt the pure bottom-up way to establish the knowledge propagation model.

Based on the natural division of complex objective systems on certain level, Agent-based modeling can establish the corresponding Agent model. By bottom-up way and the study of individual behaviors, it establishes the systematic macromodel based on the interactions and mutual influences of Agents. The Agent-based modeling is mainly through multiagent interactions to describe the complexity of knowledge propagation network and the uncertainty of the interactions between users.

This paper uses NetLogo to establish model of the knowledge propagation in Wechat. Based on the analysis of the decision process of users in Wechat and their knowledge propagation behaviors, this paper adopts the Agent-based modeling method to establish relationship of the behavioral characteristics of users with the properties and behavioral features of Agent. By constructing hypothesis and the simple interactive rules, it explores the key factors which may influence the Wechat knowledge propagation. It is a modeling method that is mainly built on the interaction of local individuals rather than on a macro and systematic base.
3.2. Agent Model Description. The knowledge propagation in Wechat mainly includes two types of Agents: the knowledge Agent and the user Agent whose individual properties and interactive rule are defined as follows.

Definition 1. Define individual property of knowledge Agent Information-Attributes = (Id, Infor_type, Importance, Num_read, Num_retweet), where

Id: the unique identification number of the knowledge.

Infor_type: the type of knowledge, Infor_type $=0$ means the common event, Infor_type: 1 means the sudden event.

Importance: the importance of knowledge content, we use the TF-IDF value to measure.

Num_read: the number of browsing the knowledge.

Num_retweet: the number of retweeting the knowledge.

Definition 2. Define the individual property of user Agent User-Attributes = (User_id, User_type, User_activity, Jaccard_user_infor, Jaccard_user_ini, Jaccard_user_con, Status), as follows.

User_Id. The unique identification number of individual user, integer.

User_Type. The type of user, User_type $=0$ denotes the reader whose main behavior is to browse the knowledge received; 
User_type $=1$ denotes the writer whose main behavior is to release the original knowledge; User_type $=2$ denotes the editor whose main behavior is to retweet the knowledge he or she browses.

User_Activity. The user's active degree, User_activity $=0$ denotes the user is extreme inactive, User_activity $=1$ denotes the user is generally active, User_activity $=2$ denotes the user is extreme active.

Jaccard_User_Infor. The Jaccard similarity between the user's interest and the knowledge content, a real number on the interval $[0,1]$.

Jaccard_User_Ini. The Jaccard distance between the user's interest and that of the person who releases the knowledge.

Jaccard_User_Con. The Jaccard distance between the user and the nearest knowledge propagators.

Status. Describe the state of users, Status $=0$ denotes the user has not retweeted any knowledge, Status $=1$ denotes the user has retweeted some knowledge and the user has transformed from a receiver to a propagator.

The user's preference strategy is not a set rule. Sometimes even the user is unsure about his preference strategy. So we need to judge the user's preference strategy based on the user's historical behavioral information as well as his static information and therefore formulate a proper user preference decision model which can satisfy the needs of different users.

The interactions of Agents refer to any interactive behaviors of Agents in any form and in any depth. Agent is a stimulus-reflection object. However, because the behaviors and preferences of the users in Wechat are hard to describe, we can only simplify it based on certain assumptions. According to the above decision algorithm, we define the rule of Agent's retweeting behaviors as follows.

Rule 1. Definition of the rule of users' retweeting behaviors

(1) suppose $U_{i}, U_{j}$ are users Agent, user $U_{i}$ sends knowledge $K_{i}$, as a friend of $U_{i}, U_{j}$ receives the knowledge $K_{i}$, where for the nearest propagator user $U_{i}$ of the user $U_{j}$, the importance of the knowledge content is Importance $=\mathrm{TF}_{i}$;

(2) if the activity of user $U_{j}$ is User_activity $=0$, then the probability of user $U_{j}$ retweeting knowledge $K_{i}$ is $P=$ 0 ;

(3) if the activity of user $U_{j}$ is User_activity $=1 \mathrm{~V}$ User_activity $=2$, the type of the user is User_type $=0$, the type of knowledge is Infor_type $=1$, the probability of user $U_{j}$ retweeting knowledge $K_{i}$ is $P=1$;

(4) if the activity of user $U_{j}$ is User_activity $=1 \mathrm{~V}$ User_activity $=2$, and when User_type $=0 \wedge$ Infor_type $=0$, if Jaccard_user_con $>0.01$, the probability of user $U_{j}$ retweeting knowledge $K_{i}$ is $P$. Otherwise, the value of $P$ is $P=$ Jaccard_user_con $* 50$. Otherwise the value of $P$ is

$$
P=\frac{1}{3}\left(\text { Jaccard }_{\text {user }_{\text {infor }}}+\text { Jaccard }_{\text {user }_{\text {ini }}}+\text { Importance }\right) \text {. }
$$

(5) If the activity of user $U_{j}$ is User_activity $=1 \mathrm{~V}$ User_activity $=2$, when User_type $=1 \vee$ User_type $=$ 2 , the probability of user $U_{j}$ retweeting knowledge $K_{i}$ is $P=0$.

Rule 2. Definition of the revolutionary rule of knowledge propagation

(1) We use User_type to define the type of $U_{j}$, we use User_activity to define the users' activity, we use Infor_type, importance to define the state of knowledge $K_{i}$. We use Jaccard_user_infor, Jaccard_user_ini, Jaccard_user_con to define the state of user $U_{j}$ to the objective knowledge, we use rule 1 to define the probability of user $U_{j}$ retweeting the objective knowledge;

(2) when users interact $P=0$, Num $\_$read $=$Num $\_$read + 1 , Status $=0$;

(3) when users interact $P>0.50$, Num_read $=$ Num_read +1 , Num_retweet $=$ Num_retweet +1 , Status $=1$.

\section{Simulation Experiment and Result Analysis}

Based on the above design, we use the multiagent simulation platform of NetLogo to simulate the knowledge propagation in Wechat.

Algorithm 3. Users' relationship network and the initialization of individual properties of Agent

(1) first, determine the starting point. Each knowledge has a corresponding release person and initializes the individual properties of knowledge Agent;

(2) according to the relationship between the release person and the propagators at different levels based on the data of Wechat community, establish the relationship between the starting point and the first level fans. Then establish the relationship between the first level fans and their second level fans;

(3) repeat step 2, until all users have established the relationship;

(4) initialize the individual properties of users and knowledge Agent; determine the initial value of each data according to the definitions.

Algorithm 4. The knowledge propagation simulation model

(1) producing the topology structure of knowledge propagation network according to Algorithm 1;

(2) starting from the initial point, interact with all the users pointed to from the initial point. According to interactive Rule 1 to judge the probability of users to retweet knowledge. According to Rule 1 and Rule 2, update the related properties of Agent;

(3) do step 2 with all the individual fans. Update all the individual properties;

(4) for all the users who retweet knowledge, repeat step 2, 3 until satisfying the end conditions. 


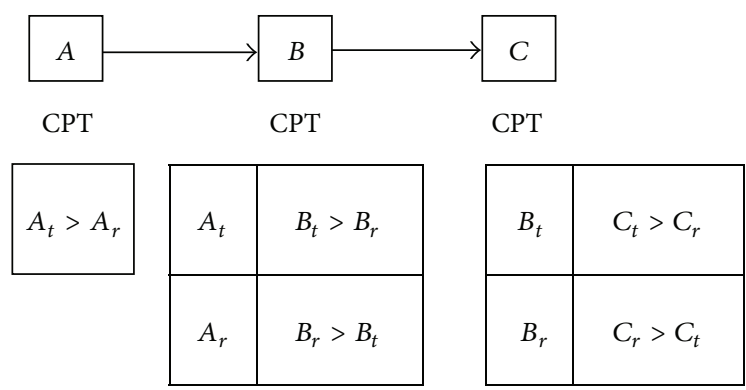

FIgURE 1: The users' preference expression map.

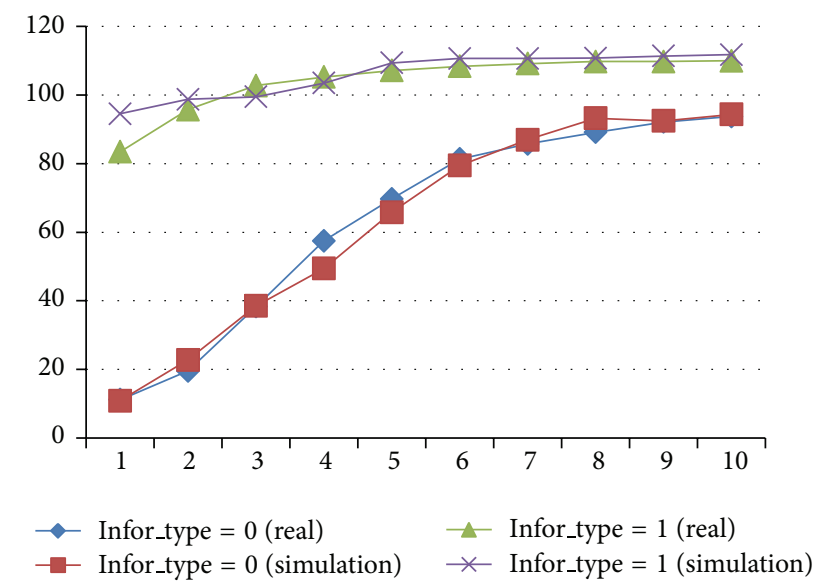

FIGURE 2: Comparison of the reading tendency of different types of knowledge.

The simulation parameters mainly include the importance of the content of objective information, the type of events, and the Jaccard similarity between the type of users and their interests. We choose 800 Wechat users in 50 undergraduate Wechat groups of Jiangxi University of Finance and Economics. We use the average value of 10 times simulations and get the results as shown in Figures 2 and 3.

The horizontal axis in Figure 1 is time with the unit of days and the vertical axis in Figure 1 is the number of reading or retweeting. We choose the health knowledge as common event and MH370 event as sudden event. From above experiment we can see the propagation rules of sudden events and common events are quite different. The sudden events have reached the peak within the first several days while the common events first propagate slowly and then become faster. However, in general the sudden events exceed the common events no matter on the number of reading or the number of retweeting. Both of the events will not arouse attentions after 10 days.

The horizontal axis in Figure 4 is the $\mathrm{TF}_{i}$ value and the vertical axis in Figure 4 is the number of reading or retweeting. We can find that when the $\mathrm{TF}_{i}$ value is bigger than 0.3 , the number of reading and retweeting has a sudden increase and the retweeting rate reaches $50 \%$ and the reading rate reaches $75 \%$. When the $\mathrm{TF}_{i}$ value continues to increase, the number of reading and retweeting will increase too

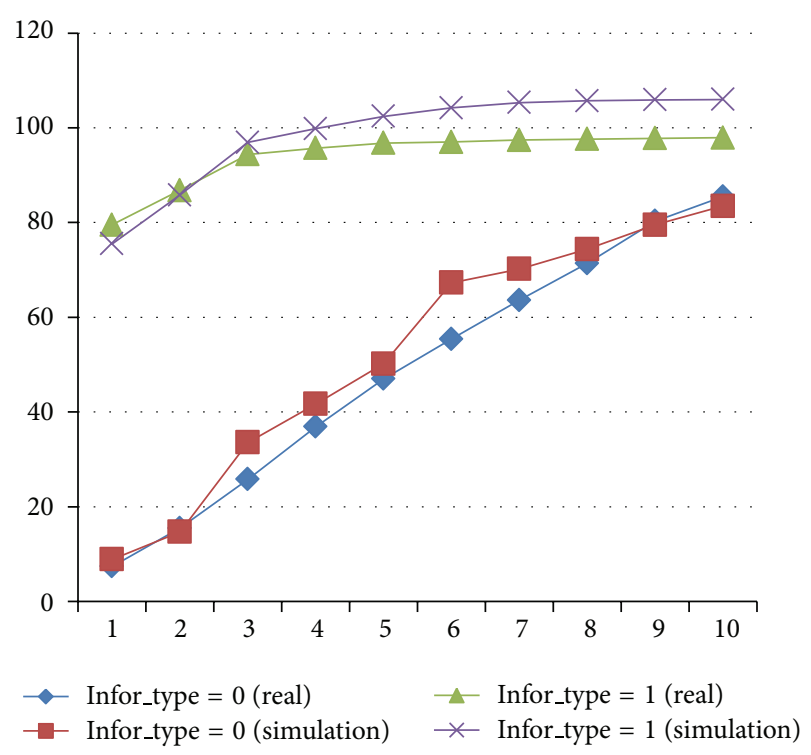

FIGURE 3: Comparison of retweeting tendency of different types of knowledge.

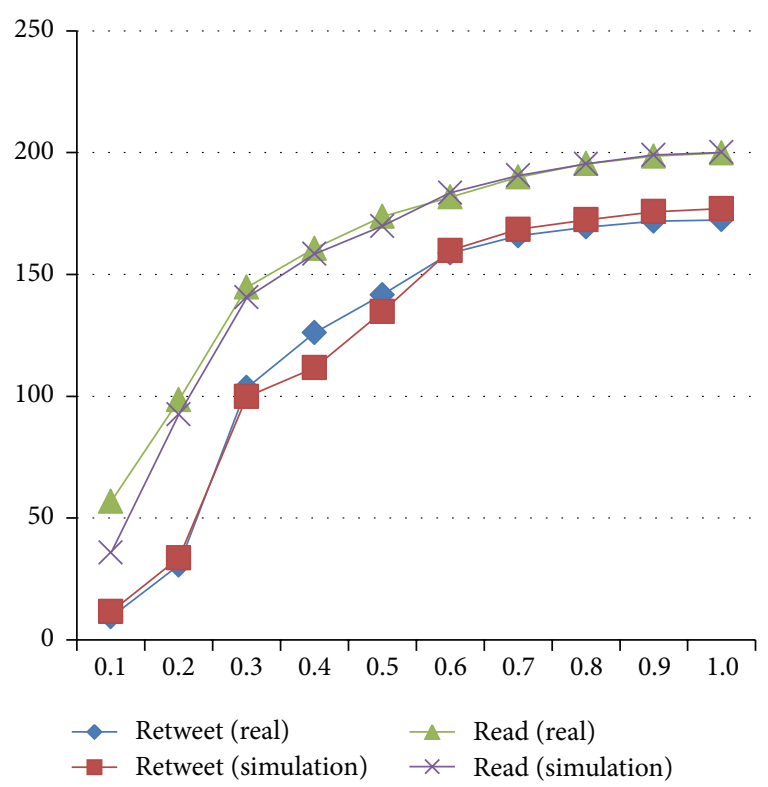

FIGURE 4: The relationship between the importance of knowledge content and propagation.

but the growth will gradually slow. When the $\mathrm{TF}_{i}$ value is approaching 1, almost all users will read and retweet the knowledge.

The horizontal axis in Figures 5, 6, and 7 is interest similarity while the vertical axis in Figures 5, 6, and 7 is the number of reading or retweeting. There is a common law in the above three figures. That is, when the similarity is bigger than 0.01 , the number of knowledge read and retweeted will increase very sharply. 


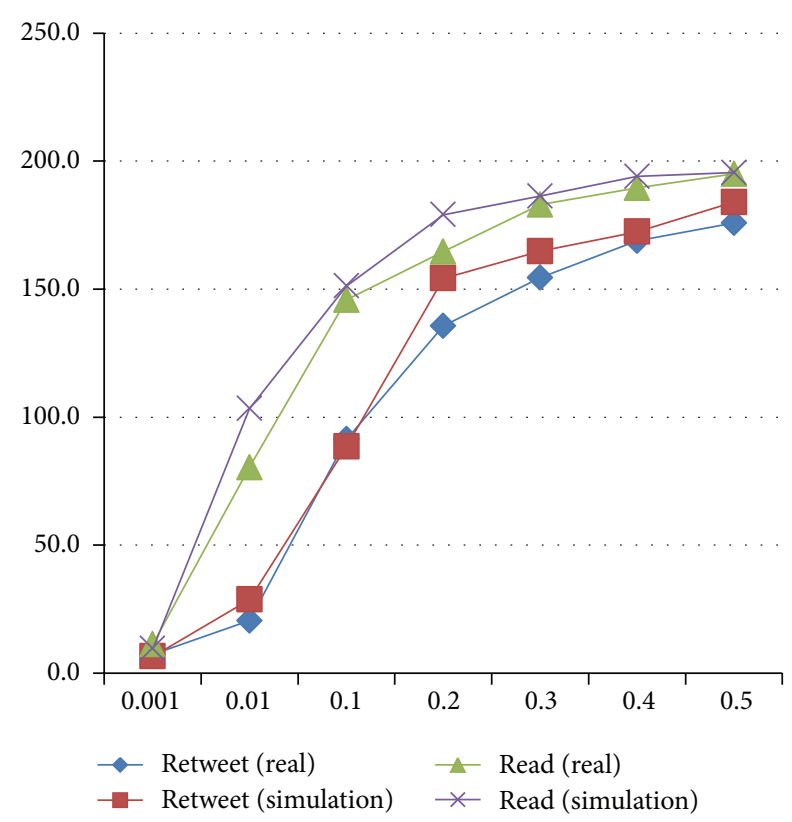

FIGURE 5: The relationship between the similarity of users' interests with the content and knowledge propagation.

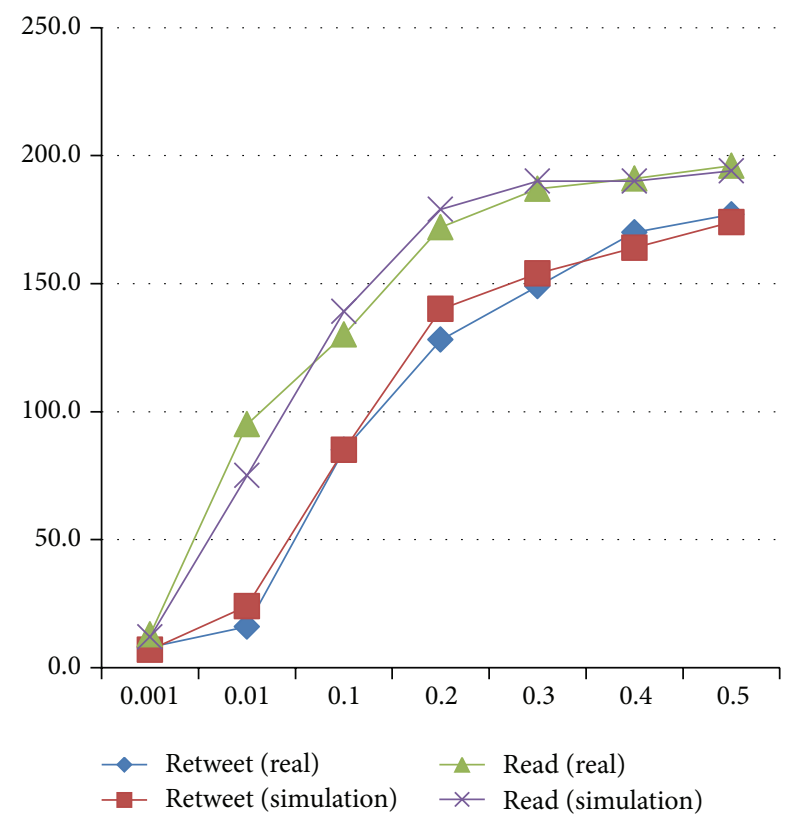

FIGURE 6: The relationship between the similarity of users' interests with the release persons' interests and knowledge propagation.

\section{Conclusions}

Nowadays, the social media have become the main carrier of knowledge propagation. Especially for youngsters, it is a kind of fashion to read and retweet knowledge through cell phones. As one of the main social media, the Wechat has only been launched for 3 years but its development is very rapidly. The Wechat network is a typical complex network. Studying

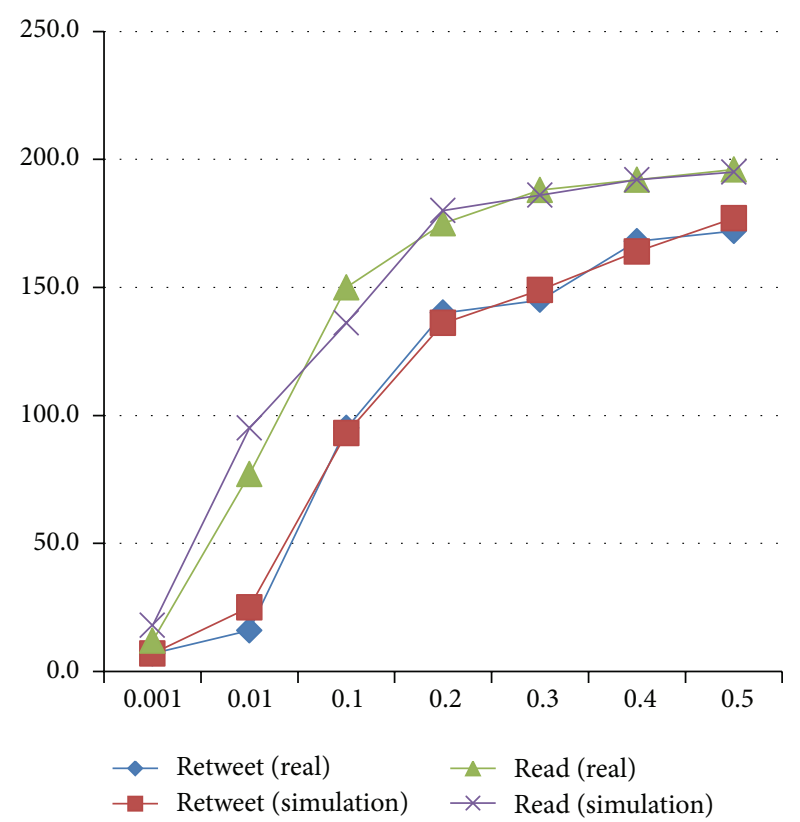

FIGURE 7: The relationship between the similarity of users' interests with the propagators' interests and knowledge propagation.

on the knowledge propagation in Wechat is an interesting and valuable work.

This paper first makes qualitative analysis on the factors of Wechat community which influence the retweeting behaviors of users and introduces the concept of preferences. By using CP-nets preference expression tool we establish the preference model and select the best feature item combination through reasoning and query way. Lastly this paper establishes the Agent knowledge propagation forecasting model. Based on the analysis of users' decisions, this paper abstracts the users in Wechat community and the knowledge propagated as Agent. By applying NetLogo and based on the "attention" relationship network between users in community, this paper constructs a knowledge propagation network according to the individual characteristics and interactive rules. This paper uses the users' historical information and their preferences to forecast the knowledge propagation tendency and simulates and analyzes the results.

We find the simulation result matches the real law of knowledge propagation, which verifies this paper's analysis on the factors influencing the knowledge propagation of Wechat users. It shows that the preferences of users are an important factor which helps users to make the decision whether to propagate the knowledge. In the experiment, we find there are some important thresholds: when the value of the importance of knowledge is bigger than 0.3 , the number of the knowledge read or retweeted will increase sharply. When the importance of knowledge continues to increase, the growth of the number of reading and retweeting will slow. When the value is bigger than 0.6 , the growth will reach the saturation state. This finding has great significance to the knowledge propagation in Wechat, which provides proof for forecasting the scale and influence of knowledge propagation. 
Considering most Wechat users are undergraduates, this paper only chooses 800 Wechat undergraduate users as experimental objects, which has certain restrictions. In the future work, we should choose the network which is more representative. Furthermore, because it has not reached the data requirement, this paper has not tested the users' preference sequences in the experiment, which can be the next step work we will take.

\section{Conflict of Interests}

The author declares no conflict of interests. The author has no financial and personal relationships with other people or organizations that can inappropriately influence the work.

\section{Acknowledgments}

This work is supported by the National Natural Science Foundation of China under Grant nos. (71361013, 71261009, 71273122, and 71163014); China Postdoctoral Science Foundation under Grant no. (2013M541867); The Education Department of Jiangxi province science and technology research projects under Grant no. (11728).

\section{References}

[1] L. Jun, Study on Employees'Knowledge Sharing Behaviors Based on Complex Social Networks, Chongqing University Press, 2013.

[2] P. F. Drucker, Management Challenges for the 21st Century, HarperCollins, 1999.

[3] S. Wei, Study on Tacit Knowledge Flow and Transform in Corporation, Harbin Institute of Technology Press, 2008.

[4] C. Liang, C. Zhong, H. Li-Chuan, G. Hao, and Z. Yin, “The measurement of employees' knowledge stock and empirical study based on social network analysis," Journal of Industrial Engineering and Engineering Management, vol. 23, no. 4, pp. 4968, 2009.

[5] W. Sroka, J. Cygler, and B. Gajdzik, "Knowledge transfer in networks. The case of steel enteprises in Poland," Metalurgija, vol. 53, no. 1, pp. 101-104, 2014.

[6] C.-S. Lu and S.-Y. Kuo, "The effects of port employees' perceptions of tacit knowledge and transaction cost on knowledge transfer," International Journal of Shipping and Transport Logistics, vol. 6, no. 1, pp. 46-68, 2014.

[7] L. Colombo and P. Labrecciosa, "Inter-firm knowledge diffusion, market power, and welfare," Journal of Evolutionary Economics, vol. 22, no. 5, pp. 1009-1027, 2012.

[8] J.-J. Huang, "Knowledge diffusion models-perspectives of gene evolution and population dynamics," Knowledge Management Research \& Practice, vol. 11, no. 3, pp. 313-322, 2013.

[9] P. E. Eriksson, "Exploration and exploitation in project-based organizations: development and diffusion of knowledge at different organizational levels in construction companies," International Journal of Project Management, vol. 31, no. 3, pp. 333341, 2013.

[10] M. H. Ho and J. S. Liu, "The motivations for knowledge transfer across borders: the diffusion of data envelopment analysis (DEA) methodology," Scientometrics, vol. 94, no. 1, pp. 397-421, 2013.
[11] I. Nonaka, R. Toyama, and A. Nagata, "A firm as a knowledgecreating entity: a new perspective on the theory of the firm," Industrial and Corporate Change, vol. 9, no. 1, pp. 1-20, 2000.

[12] F. Sabetzadeh and E. Tsui, "Social motives polarity and its impact on knowledge sharing," VINE, vol. 41, no. 1, pp. 76-88, 2011.

[13] G. Niu, "A game theory based analysis of the tacit knowledge sharing and incentive mechanism," Advanced Materials Research, vol. 601, no. 4, pp. 564-569, 2013.

[14] P. Hendriks, "Why share knowledge? the influence of ICT on the motivation for knowledge sharing," Knowledge and Process Management, vol. 6, no. 2, pp. 91-100, 1999.

[15] T. H. Davenport and L. Prusak, Working Knowledge: How Organizations Manage What They Know, Harvard Business School Press, Boston, Mass, USA, 1998.

[16] R. S. Sharma and S. Bhattacharya, "Knowledge dilemmas within organizations: resolutions from game theory," Knowledge-Based Systems, vol. 45, no. 6, pp. 100-113, 2013.

[17] K. M. Bartol, W. Liu, X. Zeng, and K. Wu, "Social exchange and knowledge sharing among knowledge workers: the moderating role of perceived job security," Management and Organization Review, vol. 5, no. 2, pp. 223-280, 2009.

[18] X. Wang, "Forming mechanisms and structures of a knowledge transfer network: theoretical and simulation research," Journal of Knowledge Management, vol. 17, no. 2, pp. 278-289, 2013.

[19] D. Chalmers, "Social innovation: an exploration of the barriers faced by innovating organizations in the social economy," Local Economy, vol. 28, no. 1, pp. 17-34, 2013.

[20] P. M. Senge, “Communities of leaders and learners," Harvard Business Review, vol. 75, no. 5, pp. 30-32, 1997.

[21] S. Wilson, O. Liber, M. Johnson, P. Beauvoir, P. Sharples, and C. Milligan, "Personal learning environments: challenging the dominant design of educational systems," Journal of e-Learning and Knowledge Society, vol. 3, no. 2, pp. 27-38, 2007.

[22] W. Lin, "Research on knowledge sharing and interpersonal relationships: empirical study of family firms and non-family firms," Quality and Quantity, vol. 47, no. 1, pp. 151-166, 2013.

[23] V. Buskens and K. Yamaguchi, "A new model for information diffusion in heterogeneous social networks," Sociological Methodology, vol. 29, no. 1, pp. 281-325, 1999.

[24] I. Z. Kiss, M. Broom, P. G. Craze, and I. Rafols, "Can epidemic models describe the diffusion of topics across disciplines?" Journal of Informetrics, vol. 4, no. 1, pp. 74-82, 2010.

[25] X.-D. Xu, Y.-T. Xiao, and S.-R. Zhu, "Simulation investigation of rumor propagation in microblogging community," Computer Engineering, vol. 37, no. 10, pp. 272-274, 2011.

[26] S. Cyr and C. W. Choo, "The individual and social dynamics of knowledge sharing: an exploratory study," Journal of Documentation, vol. 66, no. 6, pp. 824-846, 2010.

[27] C. Mitton, C. E. Adair, E. McKenzie, S. B. Patten, and B. W. Perry, "Knowledge transfer and exchange: review and synthesis of the literature," The Milbank Quarterly, vol. 85, no. 4, pp. 729-768, 2007.

[28] S. Wang and R. A. Noe, "Knowledge sharing: a review and directions for future research," Human Resource Management Review, vol. 20, no. 2, pp. 115-131, 2010.

[29] E. M. Rogers, Diffusion of Innovation, Free Press, New York, NY, USA, 2003.

[30] R. Cowan and N. Jonard, "Network structure and the diffusion of knowledge," Journal of Economic Dynamics \& Control, vol. 28, no. 8, pp. 1557-1575, 2004. 
[31] R. Cowan, N. Jonard, and J. B. Zimmermann, "Evolving networks of inventors," Journal of Evolutionary Economics, vol. 16, no. 1, pp. 155-174, 2006.

[32] P. Morone and R. Taylor, "Knowledge diffusion dynamics and network properties of face-to-face interactions," Journal of Evolutionary Economics, vol. 14, no. 3, pp. 327-351, 2004.

[33] C. Jianxun, "Studies on the Dynamics of Knowledge Communication based on Complex Networks," University of Science and Technology of China, 2006.

[34] J. Li and D. Sun, "Knowledge propagation model in complex networks," Journal of South China University of Technology, vol. 34, no. 6, pp. 99-102, 2006.

[35] H. Wanly, "The complex network model of knowledge diffusion," Operations Research and Management Science, vol. 17, no. 5, pp. 150-154, 2008.

[36] M. T. Hansen, M. L. Mors, and B. Løvås, "Knowledge sharing in organizations: multiple networks, multiple phases," Academy of Management Journal, vol. 48, no. 5, pp. 776-793, 2005.

[37] G. Lai and O. Wong, "The tie effect on information dissemination: The spread of a commercial rumor in Hong Kong," Social Networks, vol. 24, no. 1, pp. 49-75, 2002.

[38] W. Tsai, "Knowledge transfer in intraorganizational networks: effects of network position and absorptive capacity on business unit innovation and performance," Academy of Management Journal, vol. 44, no. 5, pp. 996-1004, 2001.

[39] R. Reagans and B. McEvily, "Network structure and knowledge transfer: The effects of cohesion and range," Administrative Science Quarterly, vol. 48, no. 2, pp. 240-356, 2003.

[40] Y. Jiang and J. C. Jiang, "Understanding social networks from a multiagent coordination perspective," IEEE Transactions on Parallel and Distributed Systems, no. 99, pp. 1-17, 2013.

[41] X. Xiao-dong, X. Yin-tao, and Z. Shi-rui, "Simulation investigation of rumor propagation in microblogging community," Computer Engineering, vol. 37, no. 10, pp. 272-274, 2011.

[42] Z. Yang, J. Tang, J. Li, and W. Yang, "Social community analysis via a factor graph model," IEEE Intelligent Systems, vol. 26, no. 3, pp. 58-65, 2011.

[43] J. Zeng, S. Zhang, and C. Wu, "A framework for WWW user activity analysis based on user interest," Knowledge-Based Systems, vol. 21, no. 8, pp. 905-910, 2008. 


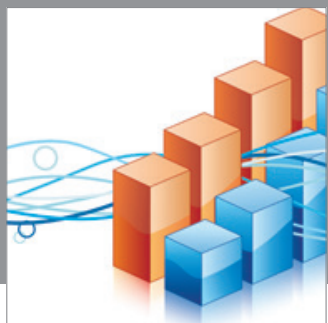

Advances in

Operations Research

mansans

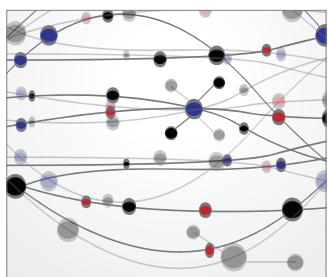

The Scientific World Journal
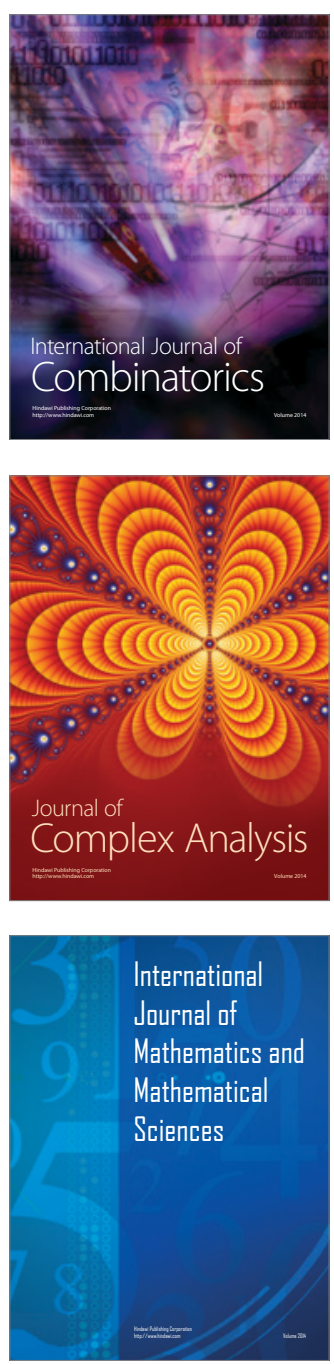
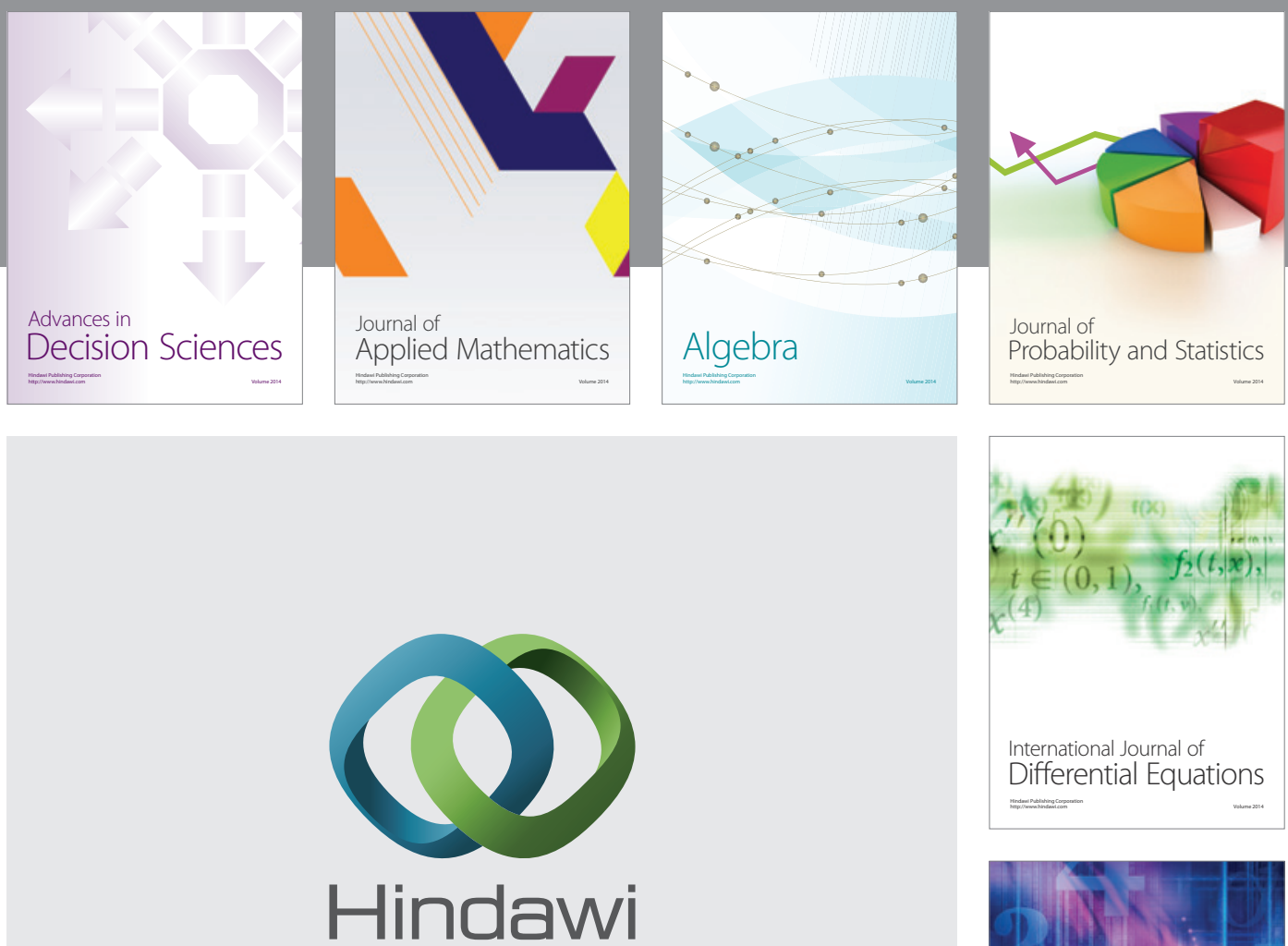

Submit your manuscripts at http://www.hindawi.com
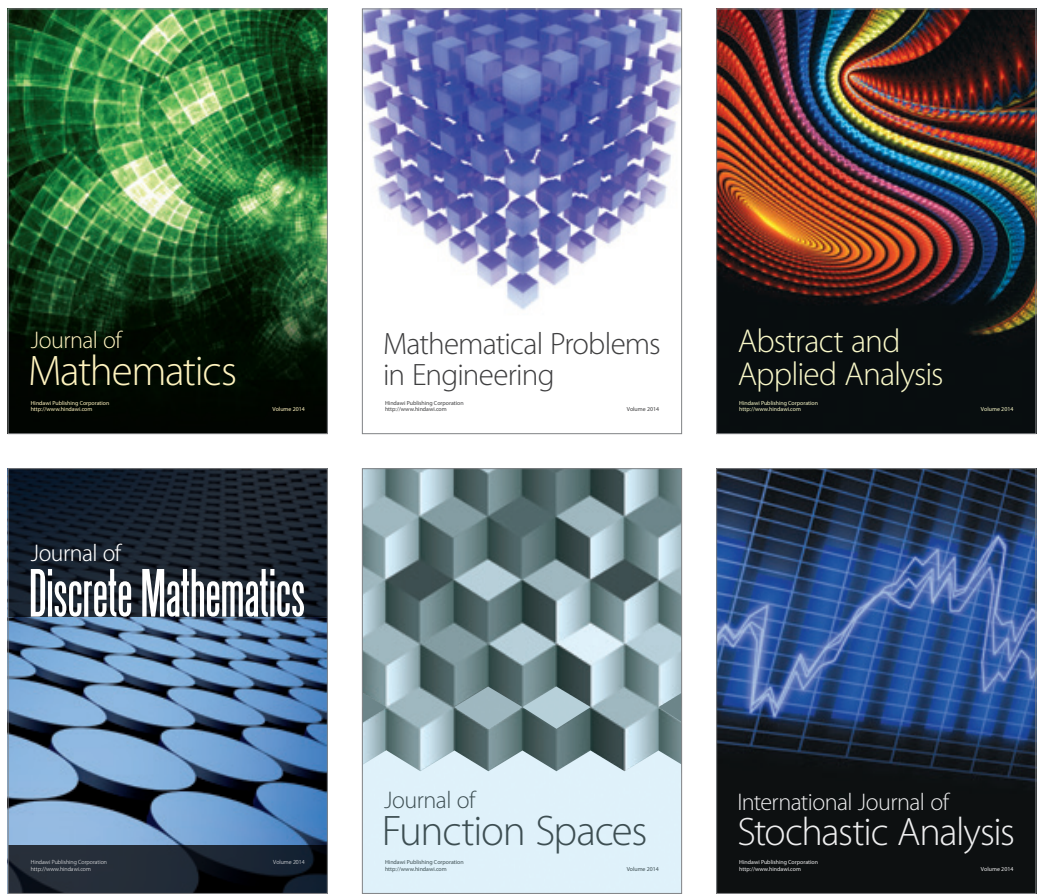

Journal of

Function Spaces

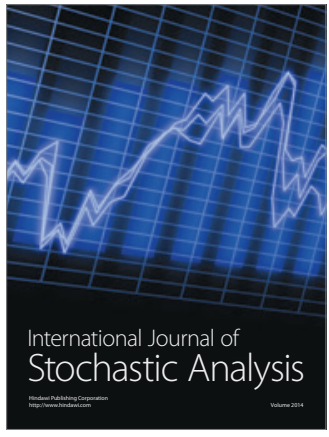

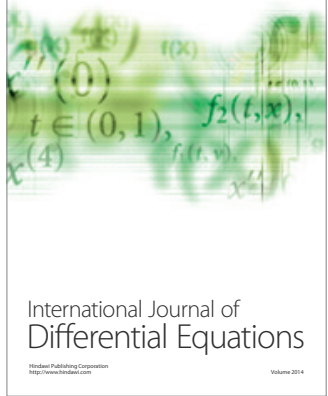
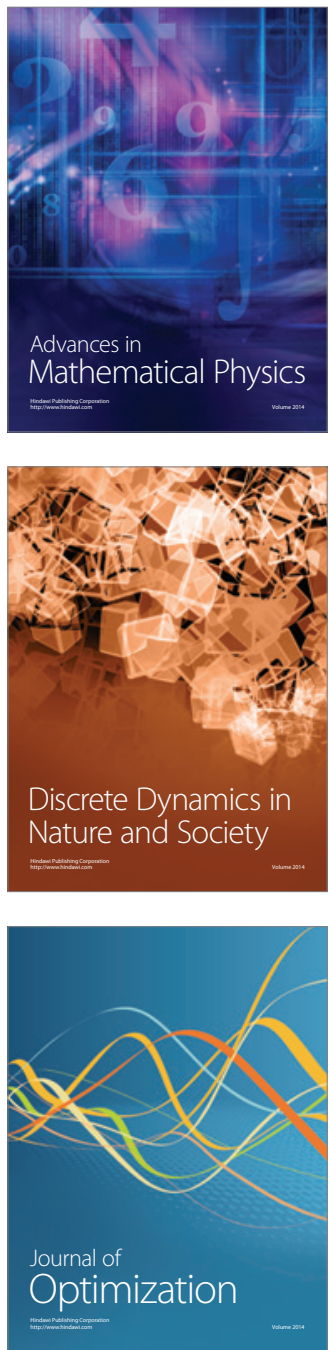